\title{
A Robust Pair Copula-Point Estimation Method for Probabilistic Small Signal Stability Analysis with Large Scale Integration of Wind Power
}

\author{
P. Kanyingi, Keyou Wang, Guojie Li, and Wei Wu
}

\begin{abstract}
In this paper, a robust Pair Copula-Point Estimation Method is proposed to probabilistically analyse the impact of wind power complex dependencies and uncertainties in both wind power and loads towards a power system's small signal stability. The proposed approach is adopted to probabilistically analyse the small signal stability of the IEEE 39-bus test system at three different wind power penetration levels concurrently considering light and heavy loading conditions. Increasing wind power penetration, uncertainties in both wind power and loads and the complex dependencies between wind power sources are probabilistically established to negatively impact the system's small signal stability. The worst case is observed when the level of wind power penetration is highest alongside consideration of the uncertainties arising from both wind power and the loads, the complex dependence existing between the wind power sources and at heavy loading. Thus, increasing penetration of uncertain and strongly dependent wind power sources and the uncertainty existing in power system loads should be intensively studied as they present risks towards a power system's small signal stability.
\end{abstract}

Index Terms-Dependence, probabilistic small signal stability analysis (PSSSA), pair copula, uncertainty.

\section{INTRODUCTION}

In recent years, global warming has become an issue of major concern mainly due to excessive pollution. Hence, countries around the globe are adopting more renewable energy sources especially wind power to address this problem. Wind power being nature dependent is characterised by uncertainty which introduces new challenges to power system operators with relation to system security and reliability. Power system loads are considered as an additional source of uncertainty mainly due to their varying nature. For the optimal operation of a power system integrated with such sources of uncertainty, an incorporation of uncertainty forecast in the system management is paramount [1].

Wind power sources located within the same geographical area are considered spatially dependent since they are influenced by the same physical phenomenon [2]. In order to make the analysis of power systems integrated with such

Manuscript received December 15, 2015; revised January 30, 2016.

P. Kanyingi is with the Department of Electrical Engineering at Shanghai Jiaotong University, Shanghai 200240, China (e-mail: pkkanyingi@gmail.com).

Keyou Wang, Guojie Li, and Wei Wu are with the Key Laboratory of Control of Power Transmission and Conversion, Ministry of Education, Department of Electrical Engineering, Shanghai Jiaotong University, Shanghai 200240, China (e-mail: wangkeyou@sjtu.edu.cn, liguojie@sjtu.edu.cn,wuweiceee@gmail.com). sources more realistic and accurate, both uncertainties in wind power and loads and the complex dependencies in wind power have to be accounted for.

The common deterministic approach has been widely applied in small signal stability analysis (SSSA) though it has been less credited due to conservativeness [3], [4]. In addition, the conventional deterministic approach fails to independently take into account the uncertainties existing in both wind power and the loads. As a consequence, numerous probabilistic approaches have been suggested to account for the uncertainties arising from the loads and wind power in SSSA. They include numerical methods such as the Monte Carlo Simulation (MCS) method [4], [5]; approximate methods such as the Point Estimation Method (PEM) [3], [6] and probabilistic collocation method (PCM) [7]; and analytical methods such as the cumulant based method [8]. The aforementioned methodologies have a major limitation in analysing power systems integrated with dependent wind power sources since they lack the ability to independently represent the complex dependencies existing between multiple wind power sources.

To account for the dependence between spatially dependent wind power sources, an assortment of dependence modeling methodologies have been proposed. They include copulae such as the Gaussian, Archimedian, and Pair in [1], [9] and [10] respectively; the normal transformation method [11] and the orthogonal transformation method [2], [3].

Wind power exhibits non normal distributions hence methods such as the Gaussian copula are not appropriate in modeling wind power sources since they are well adapted in representing normally distributed random variables. For Archimedian copulae, since copulae such as the Clayton and Gumbel only permit non-negative dependence, they are also limited in autonomously modeling wind power sources as negative dependence may result when modeling multiple wind power sources. For transformation based methods, they are considered less versatile since they over rely on the linear correlation matrix of the random variables. Due to these limitations, these methodologies are considered inflexible and do not allow for different dependency structures between pairs of random variables.

Major features such as high flexibility and applicability in formulating multivariate joint distributions which provide a means of relating multiple wind power sources make the pair copula most attractive in modeling dependent wind power sources hence its proposition in this paper. Such features are not exhibited by the other methodologies.

To analyse the impact of wind power complex 
dependencies and uncertainty in both wind power and loads towards a system's small signal stability (SSS), a potent Pair Copula-PEM probabilistic approach has been proposed. The proposed approach accounts for the dependence in multiple wind power sources and the uncertainty in both wind power and the loads. The rest of the paper is arranged as follows: A detailed description of the pair copula concept is presented in Section II. The proposed Pair Copula-PEM probabilistic approach is described in Section III with a validation of the pair copula presented in Section IV. Case studies based on the IEEE 39-bus test system are presented in Section $\mathrm{V}$ with conclusions on the findings provided in Section VI.

\section{PAIR COPULA CONSTRUCTION}

\section{A. Introduction to the Pair Copula Concept}

A Copula can be used to join different marginals together and construct new multivariate joint distributions which are instrumental in modelling dependent random variables. Considering a continuous random vector $X=\left(X_{1}, \ldots, X_{n}\right)$, any continuous random variable $X_{i}$ can be transformed to a uniform random variable $U_{i}$ and vice versa using an inverse cumulative distribution function (CDF) transformation. This is demonstrated in expression (1) as follows

$$
X_{i}=F_{i}^{-1}\left(U_{i}\right) \Leftrightarrow U_{i}=F_{i}\left(X_{i}\right)
$$

A Copula $C$ and a joint distribution of $n$ random variables can then be linked together using the Sklar's theorem as follows

$$
F\left(x_{1}, \ldots x_{n}\right)=C\left(F_{1}\left(x_{1}\right), \ldots, F_{n}\left(x_{n}\right), i=1,2, \ldots, n\right.
$$

From equation (1) and (2), a copula $C\left(u_{1}, \ldots, u_{n}\right)$ is constructed which describes the marginal distributions and their association. The copula is expressed as follows

$$
C\left(u_{1}, \ldots, u_{n}\right)=F\left(F_{1}^{-1}\left(u_{1}\right), \ldots, F_{n}^{-1}\left(u_{n}\right)\right)
$$

As highlighted in the introduction, the various types of parametric copulae lack flexibility and are limited in independently representing the complex dependencies existing between multiple wind power sources. The Pair Copula is considered more robust in representing these complex dependencies as it uses an optimal mix of the various types of parametric copulae.

\section{B. The Vine Graphical Models in Pair Copula}

In construction of the pair copula, graphical models referred to as vines are used in representing the decomposition of a joint density into a product of pair copula densities and marginal densities. The main graphical model used in representing the pair copula is referred to as the regular vine with vines such as the canonical vine (C-vine) and the drawable vine (D-vine) originating from the regular vine [12]. In terms of simplicity, the $\mathrm{C}$-vine is considered mathematically simpler when compared to the D-vine [13] hence its application in this paper. Based on the C-vine graphical model, a joint density of $n$ random variables $f\left(x_{1}, \ldots, x_{n}\right)$ can be decomposed into pair copula densities $C_{j, j+i \mid 1, \ldots, j-1}(\bullet)$ and marginals $f\left(x_{k}\right)$ as

$$
f\left(x_{1}, \ldots, x_{n}\right)=\prod_{k=1}^{n} f\left(x_{k}\right) \prod_{j=1}^{n-1} \prod_{i=1}^{n-j} C_{j, j+i \mid 1, \ldots, j-1}\left(F\left(x_{j} \mid x_{1}, \ldots, x_{j-1}\right), F\left(x_{j+i} \mid x_{1}, \ldots, x_{j-1}\right)\right)(4)
$$

Fig. 1 depicts the decomposition based on the C-vine graphical model. From the figure, it is evident that the decomposition consists of several nested trees with nodes connected using edges. Each edge corresponds to a pair copula density. For a pair copula with $n$ input random variables, the decomposition structure is made up of $n$ - 1 trees with each tree $T_{j}$ having an exclusive node that is connected to $n$ - $j$ edges. If several random variables have one random variable as the main variable such that all the other random variables are dependent on it, the $\mathrm{C}$-vine graphical model can be used to effectively represent such kind of dependence with the main variable being placed as the exclusive node in the first tree.

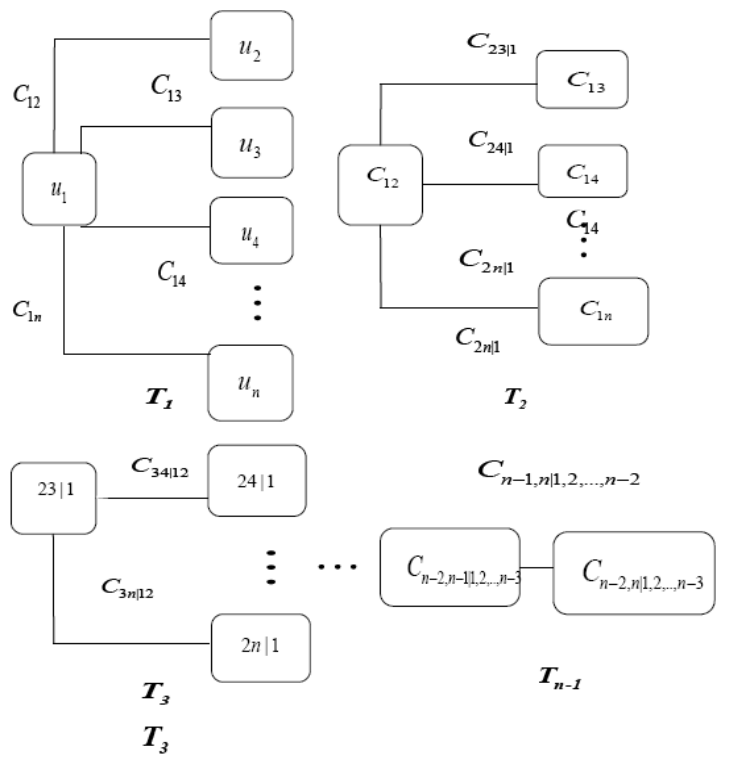

Fig. 1. The C-vine decomposition structure.

From the decomposition in expression (4), marginal conditional distributions arise which are expressed as

$$
h(a, b, \Theta)=F(a \mid b)=\frac{\partial C_{a, b}(a, b, \Theta)}{\partial b}
$$

where vectors $a$ and $b$ are uniform margins, $C_{a, b}$ is a bivariate copula CDF and $\Theta$ denotes the set of copula parameters for the copula of the joint distribution function of $a$ and $b$. Detailed analytical expressions of the function $h(\cdot)$ for different bivariate copulae are provided in [12].

\section{Simulation Procedure Using the Pair Copula Based on the C-Vine Graphical Model}

For both the C- and D-vine graphical models, random variables are sampled in the uniform domain $[0,1]$. Firstly, a 
non-parameter estimation approach is adopted to generate the marginal distributions $F_{i}$ of each wind power input in the vector $X=\left(X_{1}, \ldots, X_{n}\right)$ which are used to transform the wind power data set to the uniform domain $U=\left(U_{1}, \cdots, U_{n}\right)$. For every tree, the optimal pair copula structure is constructed after fitting parameters for the candidate copulae and selecting the optimal copula for each edge based on the least euclidean distance.

Having sampled a set of independent and uniform [0, 1] wind power data set $s=s_{i}, \ldots, s_{n}$, new sample of uniform and dependent wind power data set $v=v_{i}, \ldots, v_{n}$ is generated based on expression (6) which is later transformed back to the actual domain using the inverse CDF transformation (1).

$v_{1}=s_{1} ; v_{2}=F_{2 \mid 1}^{-1}\left(s_{2} \mid v_{1}\right), \ldots, x_{n}=F_{n \mid 1,2, \ldots, n-1}^{-1}\left(s_{n} \mid v_{1}, \ldots, v_{n-1}\right)$

The expression $F\left(s_{j} \mid v_{1}, v_{2}, \ldots, v_{j-1}\right)$ for each $j$ is evaluated using equation (5) with a general sequence expressed as

$$
F\left(s_{j} \mid v_{1}, v_{2}, \ldots, v_{j-1}\right)=\frac{\partial C_{j-1, j \mid 1, \ldots, j-2}\left(F\left(s_{j} \mid v_{1}, \ldots, v_{j-2}\right), F\left(v_{j-1} \mid v_{1, \ldots,} v_{j-2}\right)\right)}{\partial F\left(v_{j-1} \mid v_{1}, \ldots, v_{j-2}\right)}
$$

A complete summary of the whole sampling procedure is provided in algorithm 1 in the appendix.

\section{Parameter Fitting for the Appropriate Copula}

A wide range of parametric copulae is available for use in creation of an optimal pair copula structure. Parameters for the respective candidate copulae have to be fitted to allow the selection of an optimal copula for modeling a specific edge. The maximum log-likelihood estimation (8) is adopted in fitting parameters for the candidate copulae.

$$
L(l, y ; \Theta)=\sum_{e=1}^{G} \log \left(C\left(l_{e}, y_{e} ; \Theta\right)\right)
$$

where $l=\left(l_{1,1}, \ldots, l_{1, G}\right)$ and $y=\left(y_{1,1}, \ldots, y_{1, G}\right)$ are uniform wind power data vectors, $\Theta$ represents the parameters for the copula of the two data vectors and $L(l, y ; \Theta)$ is the $\log$ likelihood.

\section{E. Choice of Optimal Copulae Using Goodness of Fit Test (GOF)}

A GOF test has to be conducted to establish whether a specific edge in the $\mathrm{C}$-vine decomposition structure has been accurately modeled using a specific parametric copula. The Euclidean distance $d$ is used as a measure in choosing the best copula for each edge. The Euclidean distance is evaluated as

$$
d^{2}=\sum_{k=1}^{t}\left(C_{n}\left(u_{1 k}, \ldots, u_{n k}\right)-C_{c}\left(u_{1 k}, \ldots, u_{n k}\right)\right)^{2}
$$

where $C_{n}$ is the empirical copula of dimension $n$, which is evaluated as

$$
C_{n}\left(u_{1 k}, \ldots, u_{n k}\right)=\frac{1}{t} \sum_{k=1}^{t} I\left(x_{1 k} \leq u_{1 k}\right), \ldots, I\left(x_{n k} \leq u_{n k}\right)
$$

where $C_{c}$ is the candidate copula, $t$ is the sample size of the data set, $I$ is an indicator function such that when the conditions in the parenthesis in (10) are satisfied, it is equal to 1 otherwise it is equal to 0 .

\section{Probabilistic SSSA (PSSSA) USING THE Proposed PAIR COPULA-PEM APPROACH}

\section{A. Probabilistic Small Signal Stability Analysis (PSSSA)}

Stability in a power system is of prime importance as the system is expected to remain in a state of equilibrium under normal operating conditions or regain an equilibrium state after being subjected to disturbances such as variations in load, generation or faults [14]. The eigenvalues of the state matrix are studied to determine the time domain response of a power system to small perturbations and provide information on the stability characteristics of the system. In PSSSA, the distributions of the critical eigenvalues are established from the distribution of uncertain sources such as wind power [15] and power system loads.

The deterministic approach is undertaken iteratively to establish the probabilistic properties of the critical eigenvalues. For each critical eigenvalue, either the real part $\alpha_{r}$ or the damping ratio $\zeta_{r}$ or both are consequently analysed based on the probability indices (11) and (12) to probabilistically establish the SSS of a system. As a rule of thumb, the minimum damping ratio should be between $0.03-0.05[14]$ and the real part should be less than zero Expression (12) is used to establish the likelihoods of the system being unstable.

$$
\begin{gathered}
P\left(\alpha_{r}<0\right)=F_{\alpha_{r}}(0)=\int_{-\infty}^{0} f_{\alpha_{r}}(y) d y \\
P\left(\alpha_{r}>0\right)=1-F_{\alpha_{r}}(0)=\int_{0}^{+\infty} f_{\alpha_{r}}(y) d y \\
P\left(\zeta_{r}>0.05\right)=1-F_{\zeta_{r}}(0.05)=\int_{0.05}^{+\infty} f_{\zeta_{r}}(y) d y
\end{gathered}
$$

where $\alpha_{r}$ is the real part of the $r^{\text {th }}$ eigenvalue; $F(\cdot)$ and $f(\cdot)$ denote the CDF and the PDF of both the real part and the damping ratio; $P\left(\alpha_{r}<0\right)$ is the probability index of the real part and $P\left(\zeta_{r}>0.05\right)$ is the probability index of the damping ratio.

In this paper, the probability index (11) and (12) are adopted as they satisfactorily provide information to make conclusions on the SSS of a given test system.

\section{B. Proposed Pair Copula-PEM Approach}

With two sources of uncertainty namely wind power and the loads; and the complex dependencies existing between multiple wind power sources, the proposed approach allows for the complex dependencies and the uncertainties to be effectively captured through the pair copula and the PEM respectively. Power system loads have been previously observed to follow normal distributions. In [4] and [5], the uncertainty in loads is considered having modeled the loads 
using normal distributions hence a similar distribution is adopted in this paper. The multivariate normal distribution is adopted to generate data entries for the loads. With the generated wind power and load data set, the pair copula is initially implemented then the PEM approach based on the $2 m+1$ scheme.

In the PEM, the raw moments of each output random variable $z_{i}$ (eigenvalues) are computed from the raw moments of $n$ input random variables (wind power outputs and loads).

$$
z_{i}=F_{i}\left(x_{1}, x_{2}, \ldots, x_{m}\right)
$$

Cumulants are then established using the raw moments of the output random variable and distributions formulated using the Cornish Fisher expansion. In [11] and [16], the Cornish fisher expansion is observed to have better convergence performance as opposed to the Gram Charlier expansion hence its adoption in this paper.

In the Cornish Fisher expansion, the quantiles $(\alpha)$ of a CDF $F\left(z_{i}\right)$ are approximated in terms of the quantiles of a normal $N(0,1)$ distribution $\phi$ and the cumulants of $F\left(z_{i}\right)$. Using the first five cumulants, the Cornish Fisher expansion is expressed as

$$
\begin{aligned}
z_{i}(\alpha) & \approx \phi^{-1}(\alpha)+\frac{1}{6}\left(\phi^{-1}(\alpha)^{2}-1\right) K_{3}+\frac{1}{24}\left(\phi^{-1}(\alpha)^{3}-3 \phi^{-1}(\alpha)\right) K_{4} \\
& -\frac{1}{36}\left(2 \phi^{-1}(\alpha)^{3}-5 \phi^{-1}(\alpha)\right) K_{3}^{2}+\frac{1}{120}\left(\phi^{-1}(\alpha)^{4}-6 \phi^{-1}(\alpha)^{2}+3\right) K_{5} \\
& -\frac{1}{24}\left(\phi^{-1}(\alpha)^{4}-5 \phi^{-1}(\alpha)^{2}+2\right) K_{3} K_{4} \\
& +\frac{1}{324}\left(12 \phi^{-1}(\alpha)^{4}-53 \phi^{-1}(\alpha)^{2}+17\right) K_{3}^{3}
\end{aligned}
$$

where $z_{i}(\alpha)=F^{-1}\left(z_{i}\right)$ and $\left(K_{1,2, \ldots .5}\right)$ are the first five cumulants.

Probabilities of the eigenvalues can be obtained from the CDFs' of the critical eigenvalues. Using the probabilities, the system's SSS can be probabilistically analysed using the probability index (11) and (12). The proposed approach is summarised in the flow chart presented in Fig. 2 with detailed descriptions of the equations provided in the flow chart available in algorithm 2 in the appendix or in [17] and [18].

\section{Validation of the Results Using the MCS Method}

Due to the accuracy of the MCS method, it is mostly applied as a confirmatory method. Hence, in this study, the MCS method is adopted to confirm the results from the proposed pair copula-PEM approach with dependence between the wind power sources modeled using the pair copula approach as described in Section II and the load data generated using a multivariate normal distribution.

The conventional MCS approach is consequently applied to probabilistically establish the SSS of the system.

\section{Modeling of the Wind Farms}

Generally, wind farms consist of several wind turbines and as such, detailed modeling would result to higher order of the system model which would greatly lengthen the computational burden [19]. Hence aggregation is applied to reduce the number of wind turbines. Through aggregation, an equivalent wind turbine generator which has a power rating equal to the total of the rated power of the individual wind turbines is achieved and consequently a lower system order model is attained.

The wind farms are modeled based on the doubly fed induction generator (DFIG) wind turbines which are widely applied in modern power system because of their numerous benefits as described in [20].

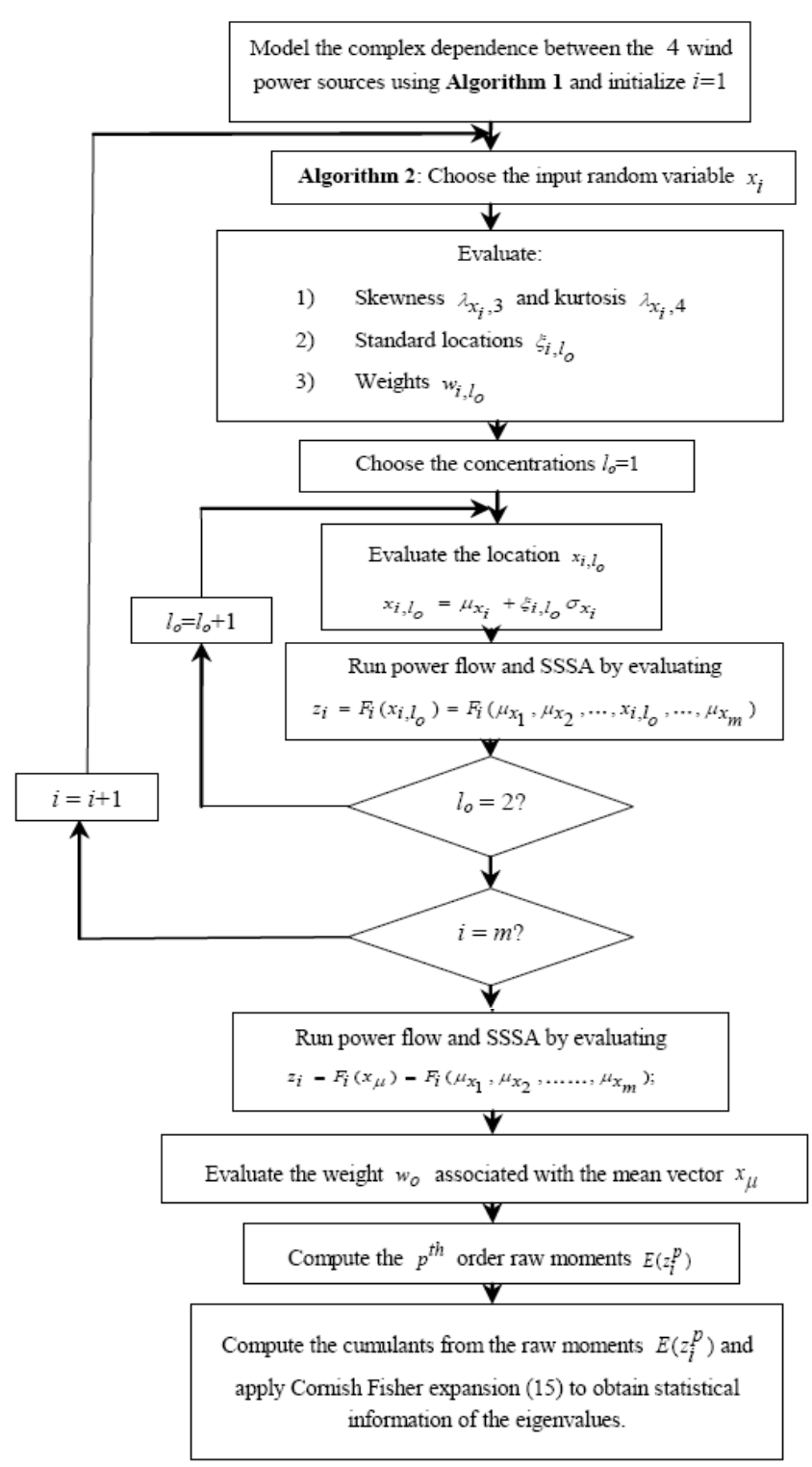

Fig. 2. Flow chart summarizing the proposed pair copula-PEM algorithm.

\section{PAIR COPUla VALidATION IN MODELING THE COMPLEX DEPENDENCE IN FOUR WIND POWER SOURCES}

\section{A. Validation of the Constructed Pair Copula Structure}

In this study, four wind power sources are considered and the complex dependence between them modeled using the pair copula algorithm. The wind power data set used in the study is obtained from the National Renewable Energy Laboratory NREL, eastern wind dataset [21].

The Euclidean distance as expressed in equation (9) is used to select the optimal copula for modeling each edge in the 
C-vine graphical decomposition. The optimal copula constructed to model the complex dependence structure between the four wind power sources is provided in Table I.

Various forms of dependence are observed between the four wind power sources with positive dependence existing between the wind power source pairs 1-4 and 2-3. This can be verified from the linear correlation coefficient matrices provided in (16).

$$
\boldsymbol{\rho}_{1,4}=\left(\begin{array}{ll}
1.0000 & 0.8804 \\
0.8804 & 1.0000
\end{array}\right) ; \boldsymbol{\rho}_{2,3}=\left(\begin{array}{ll}
1.0000 & 0.4273 \\
0.4273 & 1.0000
\end{array}\right)
$$

From Table I, the whole pair copula structure has a total of three trees since four wind power sources are considered. For the first tree $T_{1}, t$, Frank and Clayton copulae with Euclidean distances $0.3518,0.2794$ and 0.5154 have been used to model the edges $C_{12}\left(F\left(x_{1}\right), F\left(x_{2}\right)\right) \quad, \quad C_{13}\left(F\left(x_{1}\right), F\left(x_{3}\right)\right) \quad$ and $C_{14}\left(F\left(x_{1}\right), F\left(x_{4}\right)\right)$ respectively. These copulae have the shortest Euclidean distance in every row. Archimedian copulae of types Clayton and Gumbel don't permit negative dependence due to limited parameter space hence they have not been considered among the candidate copulae for modeling the edges $C_{12}\left(F\left(x_{1}\right), F\left(x_{2}\right)\right)$ and $C_{13}\left(F\left(x_{1}\right), F\left(x_{3}\right)\right)$. This can be verified from Table I since these edges correspond to the negative correlation coefficients -0.2129 and -0.3471 respectively.

A similar optimal copula selection criterion is adopted for trees $T_{2}$ and $T_{3}$ with the complete optimal pair copula structure provided in the last column of Table I. The edges in tree $T_{2}$ are modeled using Frank and Gaussian copulae and the edge in tree $T_{3}$ modeled using the Frank copula.

TABLE I: Optimal PAIR CoPUla StRUCTURe BASED ON THE Minimum EUCLIDEAN DistanCE

\begin{tabular}{ccccccccc}
\hline $\begin{array}{c}\text { Tree } \\
T_{1, \ldots, n-1}\end{array}$ & Edge & Gaussian & $\boldsymbol{t}$ & Frank & Clayton & Gumbel & $\begin{array}{c}\text { Correlation } \\
\text { coefficient }(\boldsymbol{\rho})\end{array}$ & $\begin{array}{c}\text { Optimal copula } \\
\text { structure }\end{array}$ \\
\hline & $C_{12}$ & 0.3813 & 0.3518 & 0.3704 & - & - & -0.2129 & $t$ \\
$\mathbf{1}$ & $C_{13}$ & 0.4781 & 0.3712 & 0.2794 & - & - & -0.3471 & Frank \\
& $C_{14}$ & 1.4137 & 0.9418 & 0.5932 & 0.5154 & 2.4124 & 0.8804 & Clayton \\
& $C_{23 \mid 1}$ & 0.8771 & 0.8451 & 0.6642 & 1.7517 & 0.8844 & 0.3641 & Frank \\
$\mathbf{2}$ & $C_{24 \mid 1}$ & 0.748 & 0.7481 & 0.7713 & - & - & -0.0146 & Gaussian \\
\hline \hline
\end{tabular}

\section{B. Additional Validation of the Constructed Pair Copula Structure}

An evaluation of the scatter plots of two edges $C_{13}\left(F\left(x_{1}\right), F\left(x_{3}\right)\right)$ and $C_{14}\left(F\left(x_{1}\right), F\left(x_{4}\right)\right)$ is undertaken to further demonstrate the validity of the constructed pair copula structure. The scatter plots are provided in Fig. 3.

The Frank copula is symmetric in nature with the line of symmetry occurring either along the main or the reverse diagonal. In Fig. 3(a), a concentration of mass occurs along the reverse diagonal which affirms the negative correlation coefficient of -0.3471 provided in Table I. Symmetry occurs along the same diagonal which confirms that the Frank copula is the most appropriate copula in modeling the edge $C_{13}\left(F\left(x_{1}\right), F\left(x_{3}\right)\right)$.

The Clayton copula in normally characterised by high negative tail dependence. From Fig. 3(b), a concentration of mass occurs along the main diagonal which affirms the positive correlation coefficient of 0.8804 presented in Table I and shows that positive dependence exists between the pair 1-4. The occurrence of high negative tail dependence affirms that the Clayton copula is the best copula for modeling the edge $C_{14}\left(F\left(x_{1}\right), F\left(x_{4}\right)\right)$.
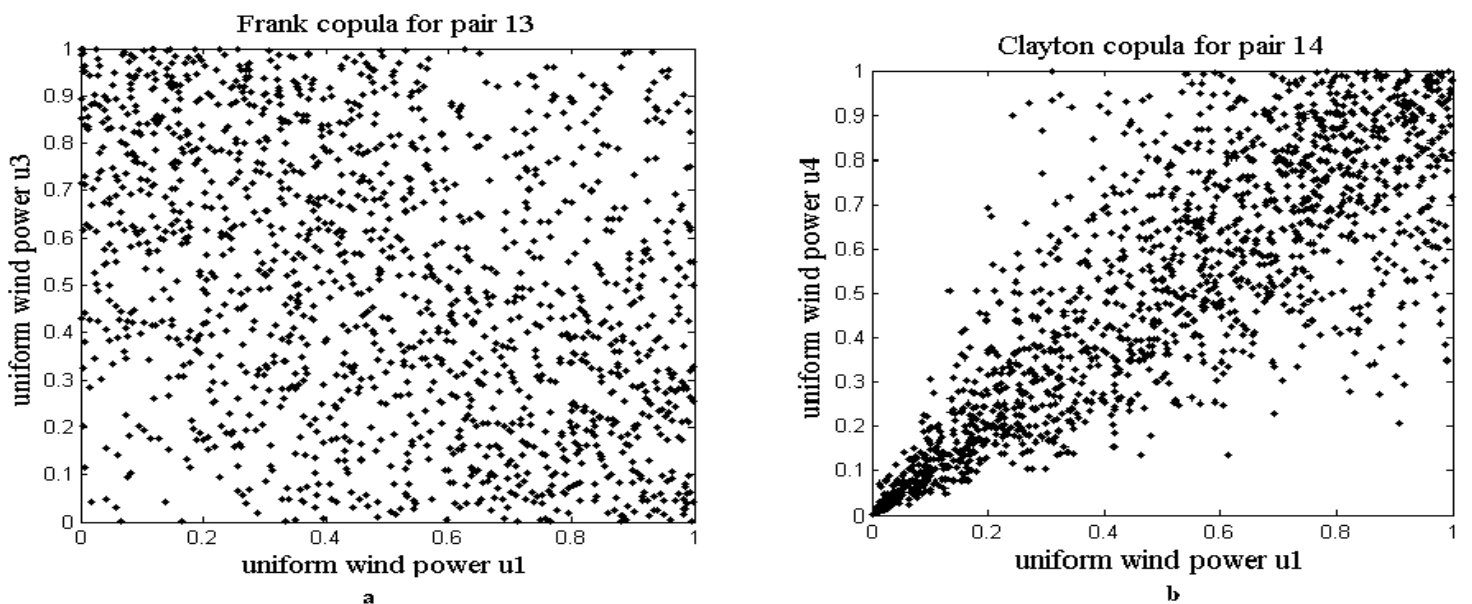

Fig. 3. Scatter plots (a) for uniform wind power sources 1-3 and (b) for uniform wind power sources 1-4. 
TABLE II: TREND OF THE CRITICAL OSCILLATORY MODE FOR CASES A AND B

\begin{tabular}{|c|c|c|c|c|}
\hline \multicolumn{5}{|c|}{ CASE A } \\
\hline \multirow[t]{2}{*}{ WPP (\%) } & \multirow[t]{2}{*}{ CRITICAL OSCILLATORY MODE } & \multirow[t]{2}{*}{ DAMPING RATIO } & \multicolumn{2}{|c|}{ PROBABILITY (\%) } \\
\hline & & & PAIR-PEM & MCS \\
\hline 10 & $-0.14106 \pm 1.3926 \mathrm{i}$ & 0.10129 & 95.47 & 95.83 \\
\hline 20 & $-0.08999 \pm 1.6157 \mathrm{i}$ & 0.05532 & 94.97 & 94.95 \\
\hline 30 & $-0.04601 \pm 1.9067 i$ & 0.02413 & 92.96 & 92.41 \\
\hline \multicolumn{5}{|c|}{ CASE B } \\
\hline \multirow[t]{2}{*}{ WPP (\%) } & \multirow[t]{2}{*}{ CRITICAL OSCILLATORY MODE } & \multirow[t]{2}{*}{ DAMPING RATIO } & \multicolumn{2}{|c|}{ PROBABILITY (\%) } \\
\hline & & & PAIR-PEM & MCS \\
\hline 10 & $-0.1409 \pm 1.3574 \mathrm{i}$ & 0.10325 & 92.96 & 92.93 \\
\hline 20 & $-0.0864 \pm 1.5797 \mathrm{i}$ & 0.054612 & 89.95 & 89.77 \\
\hline 30 & $-0.0441 \pm 1.8605 \mathrm{i}$ & 0.02369 & 88.44 & 88.4 \\
\hline
\end{tabular}

\section{Comparison of the Pair Copula with Multivariate Copulae}

Archimedian copulae are limited to application in bivariate cases as they present difficulties when extended to multivariate cases [10].

As opposed to archimedian copula, elliptical copulae are easily extendable to multivariate cases hence a comparison between the pair copula and elliptical copulae is conducted to validate the flexibility of the pair copula in complex wind power dependence modelling based on the Euclidean distance.

From Table III, the pair copula is observed to be the optimal copula in modelling the dependence between the four wind power sources since it has the shortest Euclidean distance of 0.3279 .

TABLE III: A COMPARISON BETWEEN THE PAIR, GAUSSIAN AND $T$ COPULAE

\begin{tabular}{cccc}
\hline \hline & Pair & Gaussian & $\boldsymbol{t}$ \\
\hline $\begin{array}{c}\text { Euclidean } \\
\text { distance }\end{array}$ & 0.3279 & 0.4292 & 0.4253 \\
\hline \hline
\end{tabular}

\section{CASE STUdies}

In this paper, the IEEE 39-bus test system is adopted to study the impact of wind power dependence and uncertainty in both wind power and loads towards a power system's SSS. The system is modelled using the power system analysis toolbox (PSAT) in conjunction with Matlab. Four wind farms are established at buses 32, 33, 34 and 36 by displacing four synchronous generators connected to the same buses.

Two main loading conditions are considered namely light and heavy loading. At each loading condition, three cases are analysed. Cases A to $\mathrm{C}$ being for the light loading case and cases $\mathrm{D}$ to $\mathrm{F}$ being for the heavy loading condition. The cases are given acronyms as follows: Cases $\mathrm{A}$ and $\mathrm{D}$ where the uncertainty in wind power (UW) is the main factor considered Cases $\mathrm{B}$ and $\mathrm{E}$ where both uncertainty in wind power and loads (UWL) are considered and Cases $\mathrm{C}$ and $\mathrm{F}$ where wind power dependence and uncertainty in both wind power and loads (WDUWL) are considered. Wind power penetration (WPP) is varied from $10 \%, 20 \%$ to $30 \%$. In each case, the critical oscillatory modes are studied to probabilistically establish the SSS of the test system.

The probabilities presented in Tables II, IV, V and VI represent the likelihoods of the system being small signal stable with the likelihoods of the system being unstable obtained using equation (12).

\section{A. PSSSA at Light Loading Condition}

1) Considering uncertainty in wind power (Case A) and uncertainty in both wind power and loads (Case B)

For cases A and B, PSSSA is undertaken at light loading condition with the oscillatory mode presented in Table II representing the mean values of the critical mode. The respective probabilities are obtained from the CDF plots of the real part of the eigenvalue and the results affirmed using the traditional MCS method. CDF plots for the 10\% WPP case for cases A and B are presented in Fig. 4.

From Table II, a study of the eigenvalues reveals that increasing WPP makes the system tend towards small signal instability since the eigenvalue moves closer and closer towards the imaginary axis with increasing WPP. Deterministically, the system is considered small signal stable but a study of the probabilities reveals that the system has likelihoods of being unstable. For case A, with, increasing WPP, the probabilities of the system being small signal stable steadily decline from $95.47 \%$ to $92.96 \%$ with likelihoods of $4.53 \%$ and $7.04 \%$ of being unstable respectively. This can be attributed to the uncertainty arising from the wind power sources.

In case $\mathrm{B}$, a similar phenomenon is observed as that observed in case A though the probabilities of the system being stable are lower than those of case A. The probabilities decline steadily from $92.96 \%$ to $88.44 \%$ with likelihoods of $7.04 \%$ and $11.56 \%$ of being unstable respectively. Hence, due the uncertainties arising from both wind power and the loads, the system is at a greater risk of being unstable probabilistically.

2) Considering wind power dependence and uncertainty in both wind power and loads (Case C)

For case $\mathrm{C}$, the complex dependence existing between the four wind power sources is additionally considered and the probabilities of the system being stable presented in Table IV. The complex dependence is observed to increase the risk of the system being unstable even further. This can be verified by comparing the probabilities from case $\mathrm{C}$ to those from case B. For the 10\%, 20\% and 30\% WPP cases, the probabilities decrease from $92.96 \%, 89.95 \%$ and 88.44 in case $\mathrm{B}$ to $86.93 \%, 83.42 \%$ and $80.90 \%$ in case $\mathrm{C}$ respectively. This implies that the complex dependence in wind power sources increases the likelihood of the system being unstable with the highest likelihood of $19.1 \%$ occurring at the $30 \%$ WPP level. 

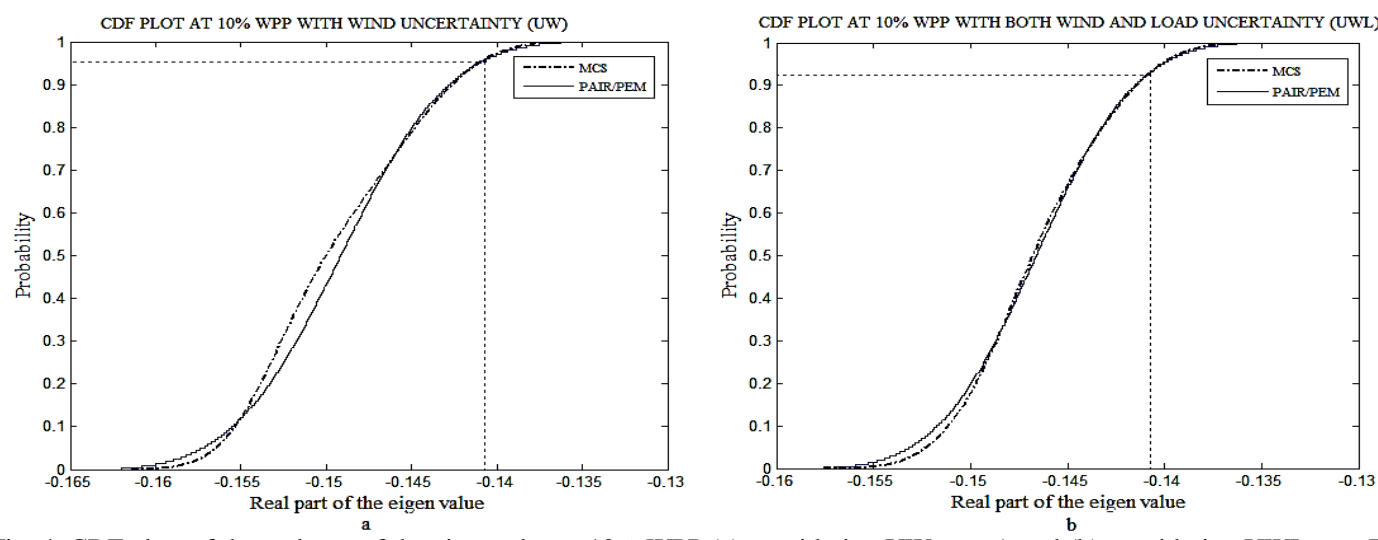

Fig. 4. CDF plots of the real part of the eigenvalue at 10\% WPP (a) considering UW case A and (b) considering UWL case B.

TABLE IV: TREND OF THE CRITICAL OSCILLATORY MODE FOR CASE C

\begin{tabular}{cccc}
\hline & & CASE C & \\
\hline WPP (\%) & CRITICAL OSCILLATORY MODE & DAMPING RATIO & \multicolumn{2}{c}{ PROBABILITY (\%) } \\
& & & 86.93 \\
PAIR-PEM
\end{tabular}

\section{B. PSSSA at Heavy Loading Condition}

1) Considering uncertainty in wind power (Case D) and uncertainty in both wind power and loads (Case E)

For cases D and E, PSSSA is undertaken at heavy loading condition with the mode presented in Table $\mathrm{V}$ representing the mean values of the system's critical mode. A study of the mode's damping ratio reveals that increased loading reduces the damping performance of the test system. This can be verified from Table $\mathrm{V}$ since the damping ratios are below the acceptable threshold range of 0.03-0.05. Similar to the light loading case, increasing WPP is also observed to move the eigenvalue closer to the imaginary axis.

The probabilities presented in Table $\mathrm{V}$ are also obtained from the CDF plots of the real part of the critical mode. CDF plots for the 10\% WPP case for cases D and E are presented in Fig. 5.

A comparison between the probabilities in case $\mathrm{D}$ and those from case $E$ reveals that high loading coupled with uncertainty arising from both wind power and the loads further decreases the probabilities of the system being stable. This can be established from the probabilities in cases D and $\mathrm{E}$ which are much lower than those from cases A and B.

The negative impact due to the uncertainty arising from the both wind power and the load is further verified in cases D and E. For case D, heavy loading coupled with uncertainty

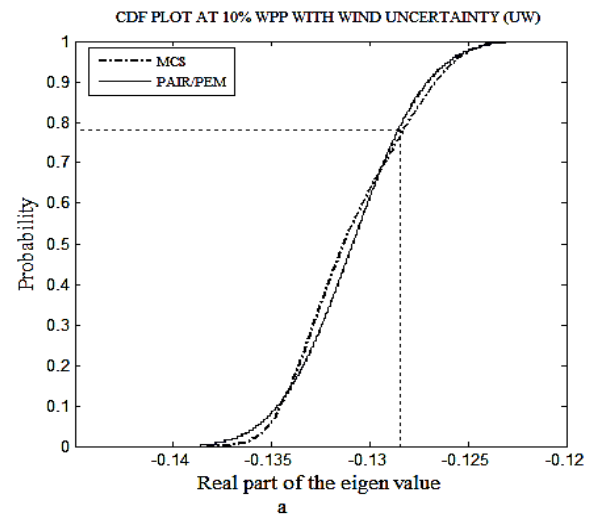

arising from wind power results to probabilities of $78.39 \%$, $75.38 \%$ and $72.36 \%$ at $10 \%, 20 \%$ and $30 \%$ WPP respectively which are much lower than those of $95.47 \%, 94.97 \%$ and $92.96 \%$ from case A hence higher likelihoods of the system being small signal unstable. A similar observation is made by comparing the probabilities in case $\mathrm{E}$ to those from case B.

Higher levels of uncertainty are also observed to increase the risk of the system being unstable since for every level of WPP in case E, lower probabilities are obtained at similar levels of WPP in case D.

2) Considering wind power dependence and uncertainty in both wind power and loads (Case F)

For case F, the complex dependence existing between the four wind power sources and high loading coupled with uncertainty arising from both wind power and the loads are observed to have the highest risk of the system being probabilistically unstable due to the lowest probabilities obtained. This can be verified from Table VI.

A comparison between the probabilities in case $\mathrm{F}$ to those from case E shows that at $10 \%, 20 \%$ and $30 \%$ WPP cases, the probabilities decrease from $75.88 \%, 73.87 \%$ and 69.36 in case $\mathrm{E}$ to $68.84 \%, 58.79 \%$ and $53.27 \%$ in case $\mathrm{F}$ respectively.

This affirms that that the complex dependence in wind power sources increases the likelihood of the system being unstable with the highest likelihood of $46.73 \%$ occurring at the $30 \%$ WPP level.

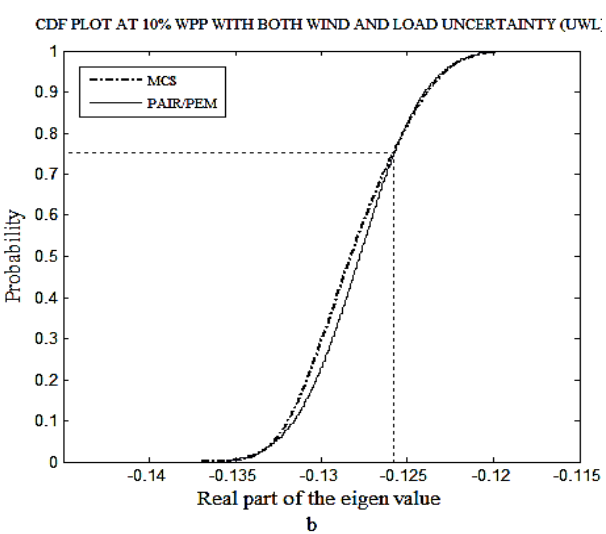

Fig. 5. CDF plot of the real part of the eigenvalue at 10\%WPP (a) considering UW case D and (b) considering UWL case E. 
TABLE V: TREND OF THE CRITICAL OSCILlatory MODE FOR CASES D AND E

\begin{tabular}{cccc}
\hline \hline & & CASE D & \\
\hline WPP (\%) & CRITICAL OSCILLATORY MODE & DAMPING RATIO & \multicolumn{2}{c}{ PROBABILITY (\%) } \\
& & & 78.39 \\
PAIR-PEM
\end{tabular}

TABLE VI: TREND OF THE CRITICAL OSCILLATORY MODE FOR CASE F

\begin{tabular}{cccc}
\hline & & CASE F & \\
\hline WPP (\%) & CRITICAL OSCILLATORY MODE & DAMPING RATIO & \multicolumn{2}{c}{ PROBABILITY (\%) } \\
PAIR-PEM & & 68.84 \\
\hline $\mathbf{1 0}$ & $-0.1109 \pm 4.6822 \mathrm{i}$ & 0.02367 & 58.79 \\
$\mathbf{2 0}$ & $-0.0992 \pm 4.7019 \mathrm{i}$ & 0.02109 & 58.98 \\
$\mathbf{3 0}$ & $-0.0264 \pm 4.9255 \mathrm{i}$ & 0.0053597 & 53.27 \\
\hline \hline
\end{tabular}
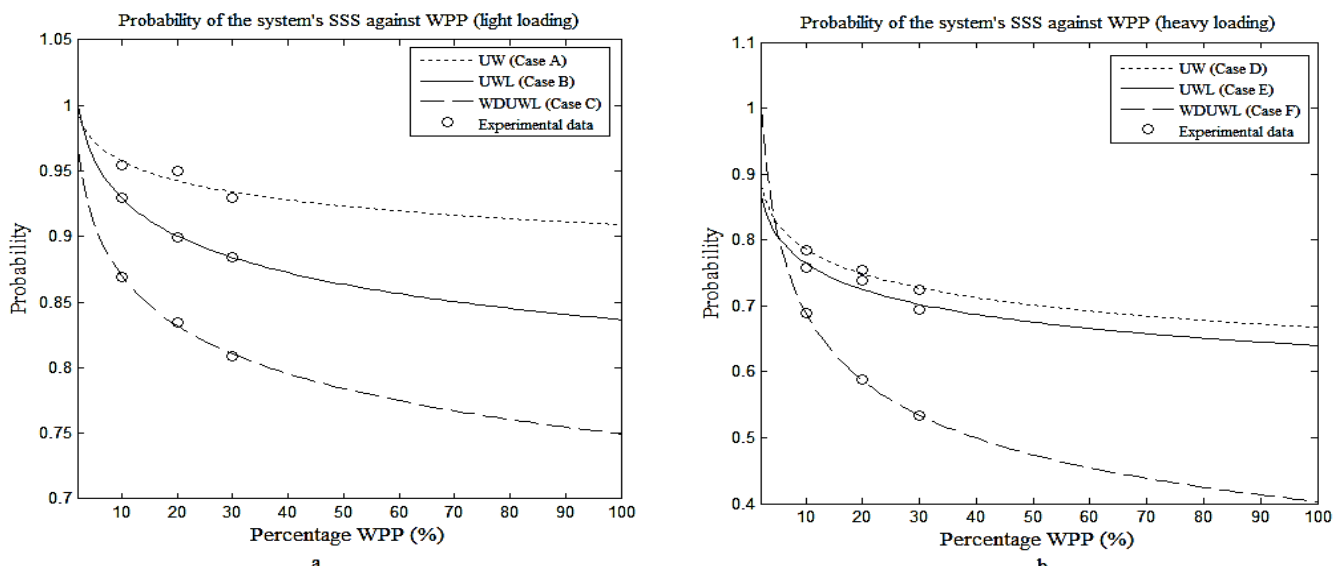

Fig. 6. Plots of probability of the system's SSS against WPP (a) at light loading and (b) at heavy loading.

\section{Projection of Probabilities for Any Level of WPP}

A projection of all the cases $A$ to $F$ is undertaken to predict the probabilities of the system being stable at different levels of WPP. Fig. 6 presents a projection of probabilities for both high and low WPP levels.

From the projection curves, for any level of WPP, lower probabilities of the system being small signal stable will be obtained at heavy loading than at light loading. For instance, for cases $\mathrm{C}$ (light loading) and $\mathrm{F}$ (heavy loading), at 40\%WPP the test system has probabilities of $79.54 \%$ and $49.9 \%$ of being stable respectively. Hence with the projection curves, it is possible to obtain the probability of the system being stable at varying levels of WPP.

\section{CONCLUSIONS}

With high dimensional uncertainties and the complex dependencies existing in modern power systems, the proposed approach provides a flexible probability model for effectively accounting for these factors in power system analysis. To improve on the proposed approach, different forms of complex dependence either positive or negative can be considered to establish which type has the worst impact towards a system's small signal stability. In addition, an advancement of studies in the time domain is critical so as to establish the transient stability of the system's under test.

\section{APPENDIX}

\section{Algorithm 1: Pair copula construction and sampling} algorithm

Construction of the optimal pair copula structure

for $i=1, \cdots, n$

set $q_{0 i}=u_{i}$

end for

for $j=1, \cdots, n-1$ do

for $w=1, \cdots, n-j d o$

For each edge $\left(q_{j-l, j}, q_{j-l, j+w}\right)$ in tree $j$

1) Fit the parameters for the respective candidate copulae using (8)

2) Select the best copula to model each edge $w$ based on the least euclidean distance (9)

3) Establish the conditional distributions $\left(q_{j, j+1}\right)$ needed for the next tree $j+1(5)$ and (7)

end for

end for 
Sampling using the optimal pair copula structure

Generate $s_{i} ; \quad i=1,2,3, \ldots, n$ independent and $U(0,1)$

$$
\begin{aligned}
& \text { Set } v_{1}=q_{1,1}=s_{1} \\
& \text { for } i=2 \text { to } n \quad \text { step }++1 \text { do } \\
& q_{i, 1}=s_{i} \\
& \text { for } \quad r=i-1 \text { to } 1 \text { step }--1 \text { do } \\
& q_{i, 1}=h^{-1}\left(q_{i, 1}, q_{r, r}, \Theta_{r, i-r}\right) \\
& \text { end for } \\
& \text { Set } v_{i}=q_{i, 1} \\
& \text { if } i=n \quad \text { then } \\
& \text { stop } \\
& \text { end if } \\
& \text { for } j=1 \text { to } i-1 \text { step }++1 \text { do } \\
& q_{i, j+1}=h\left(q_{i, j}, q_{j, j}, \Theta_{j, i-j}\right) \\
& \text { end for }
\end{aligned}
$$

end for

Transform $v_{1}, \ldots, v_{n}$ back to the actual domain by applying inverse CDF (1)

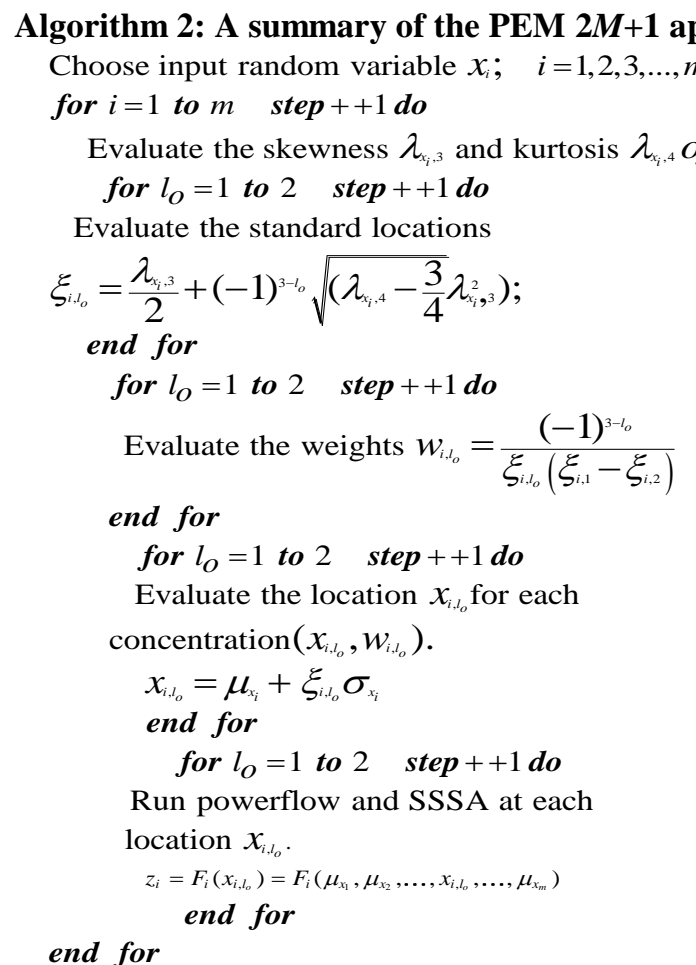

Run powerflow and SSSA at a point constituting the mean of all wind power outputs.

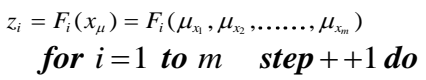

Evaluate the weight $w_{o}$ associated with the mean vector $x_{\mu}$

$$
\begin{aligned}
& w_{o}=1-\sum_{i}^{m} \frac{1}{\left(\lambda_{x_{i}, 4}-\lambda_{x_{i}, 3}^{2}\right)} \\
& \text { end for }
\end{aligned}
$$

Evaluate the $p^{\text {th }}$ order raw moment $E\left(z_{i}^{p}\right)$ of the real part of the eigenvalues.

$E\left(z_{i}^{p}\right)=\left\{\sum_{i=1}^{m} \sum_{l_{o}}^{2} w_{i, l_{o}}\left(F_{i}\left(x_{i, t_{o}}\right)\right)^{p}\right\}+w_{o}\left(F_{i}\left(x_{\mu}\right)\right)^{p}$

Evaluate the cumulants using $E\left(z_{i}^{p}\right)$.

Apply Cornish Fisher expansion (15) to obtain the statistical information of the eigenvalues. break

\section{ACKNOWLEDGMENT}

P. Kanyingi thanks Prof. Wang and Prof. Li for the support provided during the research process. Their level of dedication for the whole research process was extraordinary and worthy of credit. This work was supported in part by the Chinese government through the Chinese Scholarship Council (CSC), the National Natural Science Foundation of China under Grant (51307107, 51477098), and the National High-Tech R\&D Program of China 863 program under Grant (2015BAA01B02).

\section{REFERENCES}

[1] G. Papaefthymiou and D. Kurowicka, "Using copulas for modelling stochastic dependence in power system analysis," IEEE Trans. Power Syst., vol. 24, no. 1, pp. 40-49, Feb. 2009.

[2] J. Morales, L. Baringo, A. Conejo, and R. Minguez, "Probabilistic power flow with correlated wind sources," IET Gener. Transm. Distrib., vol. 4, no. 5, pp. 641-651, 2010.

[3] H. Yue, G. Li, and M. Zhou, "A probabilistic approach to small signal stability analysis of power systems with correlated wind sources," JEET, vol. 8, no. 6, pp. 1605-1614, 2013.

[4] J. Rueda, D. Colome, and I. Erlich, "Assessment and enhancement of small signal stability considering uncertainties," IEEE Trans. Power Syst., vol. 24, no. 1, pp. 198-207, 2009.

[5] Z. Xu, Z. Dong, and P. Zhang, "Probabilistic small signal stability analysis using monte carlo simulation," in Proc. Power and Energy Soc. Gen. Meeting IEEE, June 2005, pp. 1658-1664.

[6] M. Mohammadi and N. Soleimanpour, "Probabilistic small signal stability analysis considering wind energy," in Proc. Iranian Conf. on Smart Grid (ICSG) IEEE, May 2012, pp. 1-6.

[7] L. Meiyan, M. Jin, and Z. Dong, "Uncertainty analysis of load models in small signal stability," in Proc. Sustain. Power and Gen. Supply Conf. IEEE, April 2009, pp. 1-6.

[8] S. Bu, W. Du, H. Wang, Z. Chen, L. Y. Xiao, and H. F. Li, "Probabilistic analysis of small signal stability of large scale power systems as affected by penetration of wind generation," IEEE Trans. Power Syst., vol. 27, no. 2, pp. 762-770, 2012.

[9] H. Louie, "Evaluating archimedian copula models of wind speed for wind power modeling," in Proc. IEEE Power Eng. Soc. Conf. and Expos., July 2012, pp. 1-5.

[10] L. Qiuyu, H. Wei, Y. Min, F. Yuan, and Z. Gao, "Wind power uncertainty modeling considering spatial dependence based on pair copula theory," in Proc. IEEE PES Gen. Meet. Conf. and Expos., July 2014, pp. 1-5.

[11] J. Usaola, "Probabilistic load flow with correlated wind power injections," Electr. Power Syst. Res., vol. 80, pp. 528-536, 2010.

[12] Pair-copula constructions of multiple dependence. [Online]. Available: http://epub.ub.unimuenchen.de/1855/1/paper_487.pdf

[13] D. Kurowicka and H. Joe, Dependence Modelling Vine Copula Handbook, Singapore: World Scientific Publishing, 2011.

[14] P. Kundur, K. Morrison, J. Paserba, E. Larsen, W. P. William, M. K. Donnely et al., The Electric Power Engineering Handbook, Alabama, USA: CRC Press, 2001.

[15] S. Bu, W. Du, and F. Wang, "Probabilistic analysis of small signal stability of power systems - A survey," Sustainable Power Generation and Supply IET, pp. 1-7, September 2012.

[16] J. Usaola, "Probabilistic load flow with wind production uncertainty using cumulants and cornish-fisher expansion," in Proc. the 16th Power Syst. Computation Conf., July 2008.

[17] J. Morales and J. Perez-Ruiz, "Point estimate schemes to solve the probabilistic power flow," IEEE Trans. Power Syst., vol. 22, no. 4, pp 1594-1601, 2007.

[18] C. Su, "Probabilistic load flow computation using point estimate method," Trans. Power Syst., vol. 20, no. 4, pp. 1843-1851, 2005.

[19] N. Modi and T. Saha, "Revisiting damping performance of the Queensland network under wind power penetration," in Proc. Power and Energy Soc. Gen. Meeting IEEE, July 2012, pp. 1-8.

[20] S. Yuanzhang, L. Wang, L. Guojie, and L. Jin, “ A review on analysis and control of small signal stability of power systems with large scale integration of wind power," in Proc. Int. Conf. Power Syst. Technol. IEEE, October 2010, pp. 1-6.

[21] National Renewable Energy Laboratory, Eastern Wind Dataset, on Shore Sites. [Online]. Available: https://pfs.nrel.gov/main.html 


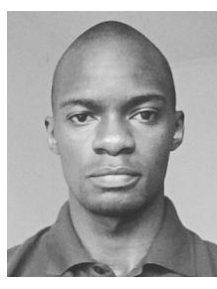

P. Kanyingi received his B.S. degree in energy engineering from Kenyatta University, Nairobi, Kenya, in 2011.

He is currently a masters student in the Department of Electrical Engineering at Shanghai Jiaotong University majoring in power systems and its automation.

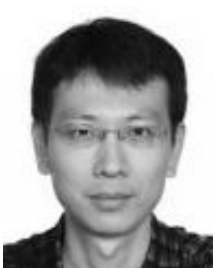

Keyou Wang received his B.S. and M.S. degrees in electrical engineering from Shanghai Jiaotong University, Shanghai, China, in 2001 and 2004, respectively, and his $\mathrm{Ph} . \mathrm{D}$. degree in electrical engineering from Missouri University of Science and Technology (formerly University of Missouri-Rolla), Rolla, MO, USA, in 2008.

He is currently an associate professor and the deputy head of the Department of Electrical Engineering at Shanghai Jiaotong University.

Prof. Keyou is a member of IEEE.

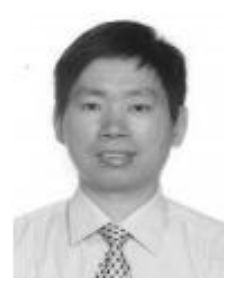

Guojie Li received his B.Eng. and M.E. degrees in electrical engineering from Tsinghua University, Beijing, China, in 1989 and 1993, respectively, and his Ph.D. degree in electrical engineering from Nanyang Technological University, Singapore, in 1999. He was an associate professor with the Department of Electrical Engineering, Tsinghua University.

$\mathrm{He}$ is currently a professor at the Department of Electrical Engineering, Shanghai Jiaotong University, Shanghai, China. Prof. $\mathrm{Li}$ is a senior member of IEEE.

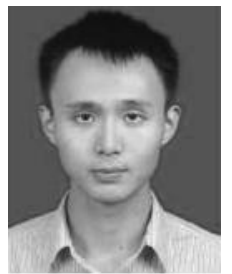

Wei Wu received his B.S. degree in electrica engineering from Huazhong University of Science and Technology, Wuhan, China, in 2012.

$\mathrm{He}$ is currently pursuing his Ph.D. degree in electrical engineering at Shanghai Jiaotong University, Shanghai, China. His research interests include power system stability and operation optimization. 